Original Article

\title{
Physical therapeutic options for residual sacrotuberous ligament pain after treatment of sacroiliac joint dysfunction
}

\author{
Takeshi Sasaki, RPT ${ }^{1)^{*}}$, Daisuke Kurosawa, MD ${ }^{1,2)}$, Eirchi Murakami, MD, $\mathrm{PhD}^{1,2)}$, \\ TAKASHI WATANABE, MD, $\mathrm{PhD}^{3)}$ \\ 1) The Japan Sacroiliac Joint and Low Back Pain Center, Japan Community Healthcare Organization \\ Sendai Hospital: 2-1-1 Murasakiyama, Izumi-ku, Sendai, Miyagi 981-3281, Japan \\ 2) Department of Orthopaedic Surgery, Japan Community Healthcare Organization Sendai Hospital, \\ Japan \\ 3) Department of Hospital Medicine, Japan Community Healthcare Organization Sendai Hospital, \\ Japan
}

\begin{abstract}
Purpose] To evaluate the incidence of pain originating from the sacrotuberous ligament after sacroiliac joint treatment, and to determine effective physical therapeutic options for sacrotuberous ligament pain. [Participants and Methods] Among 303 patients with sacroiliac joint dysfunction, 57 patients (20 males, 37 females) with persistent lower-buttock pain after sacroiliac joint injections were included in the study. The incidence of sacrotuberous ligament pain and the physical findings from the first evaluation were investigated by physical therapists. [Results] Diagnostic sacrotuberous ligament injections identified lower-buttock pain originating from the sacrotuberous ligament in $57.9 \%$ of the patients (33 out of 57 patients) after treatment of sacroiliac joint dysfunction. Of these, 11 patients experienced relief after sacrotuberous ligament injection alone; the others required physical therapy. Sacrotuberous ligament relaxation alone was effective in eight patients; biceps femoris relaxation was required in eight patients; and gluteus maximus contraction exercise was required in six patients. [Conclusion] After sacroiliac joint treatment, the incidence of residual sacrotuberous ligament pain in the persisting lower-buttock pain was $57.9 \%$. In addition to sacrotuberous ligament relaxation, biceps femoris relaxation was effective in patients who showed both higher differences in the straight leg raising test and biceps femoris tenderness, while gluteus maximus contraction exercises were effective in patients with gluteus maximus weakness.

Key words: Sacroiliac joint dysfunction, Residual sacrotuberous ligament pain, Physical therapeutic intervention
\end{abstract}

(This article was submitted Apr. 26, 2021, and was accepted Jun. 6, 2021)

\section{INTRODUCTION}

Sacroiliac joint (SIJ) dysfunction is recognized as one of the causes of lower-back and buttock pain. SIJ pain is often overlooked as it is a functional disorder that has no associated pathological conditions. Hence, it lacks specific imaging. However, clinical symptoms characteristic of patients with SIJ dysfunction are distinct from those of lumbar spine disease patients ${ }^{1)}$. Notably, pain at or around the posterior superior iliac spine (PSIS), identified as the primary pain area using patients' index finger (one-finger test) ${ }^{2}$, is the main SIJ-related symptom. SIJ pain can be diagnosed definitively after SIJ injections ${ }^{3)}$.

Tenderness in the sacrotuberous ligament (STL) (ischial tuberosity side) is one of the main physical signs of SIJ dysfunction in patients. In most patients with SIJ dysfunction, both pain at the PSIS and STL are relieved after SIJ treatments; but occasionally, lower-buttock pain originating from the STL persists even if the pain at the PSIS is relieved. Physicians and

*Corresponding author. Takeshi Sasaki (E-mail: deaiiaeb@yahoo.co.jp)

(C2021 The Society of Physical Therapy Science. Published by IPEC Inc.

(c) (i) $(-)$ This is an open-access article distributed under the terms of the Creative Commons Attribution Non-Commercial No DerivaCC BY NC ND tives (by-nc-nd) License. (CC-BY-NC-ND 4.0: https://creativecommons.org/licenses/by-nc-nd/4.0/) 
physical therapists should pay careful attention to the pain originating from the STL because it occasionally extends to the lower limbs, which mimics lumbar radiculopathy. Although, the STL has been known to cause lower-buttock pain ${ }^{4-7)}$, a method that can definitively diagnose this pain has not been established. In addition, the incidence of STL pain after treatment of SIJ dysfunctions has not been reported.

This study aimed to find the incidence of pain originating from the STL in patients with persisting lower-buttock pain after treatment for SIJ dysfunction, which was identified by administering local anesthetics to the STL, and to determine the effective physical therapeutic options for STL pain based on the physical findings from the first evaluation.

\section{PARTICIPANTS AND METHODS}

The Institutional Review Board of our hospital approved the study (Approval Number: 2020-2). Prior to the procedure, the patients provided written and informed consent for the use of their data in publications.

From January 2015 to June 2017, 303 patients were diagnosed with SIJ pain at the institution. A total of 57 patients (20 males and 37 females) with an age of $58.1 \pm 14.3$ years [mean \pm standard deviation (SD)] [range, 13-75], a height of 159.2 $\pm 10.6 \mathrm{~cm}$ [mean $\pm \mathrm{SD}$ ] [range, 144.0-185.5], and a body weight of $59.2 \pm 11.3 \mathrm{~kg}$ [mean $\pm \mathrm{SD}$ ] [range, 42.0-83.9] were included here because they suffered from lower-buttock pain that persisted even after SIJ injections.

The following items were investigated retrospectively based on medical records:

1) The incidence of lower-buttock pain originating from the STL.

2) Characteristic physical findings from the first physical examination that could yield favorable treatment results including the angle between the legs during passive straight leg raising (SLR difference), muscle tenderness of the biceps femoris muscle (BF) (1/3 distal part), and the muscle strength of the gluteus maximus muscle (GM).

3) The number of physical therapy sessions undergone and time taken to recover from the STL pain.

Diagnosis of SIJ dysfunction: All patients identified the PSIS as the main location of pain using their index finger (onefinger test $)^{2)}$ and scored more than a total of four points in the SIJ scoring system ${ }^{1)}$. The scoring system consists of six items: (1) the one-finger test, (2) groin pain, (3) pain while sitting on a chair, (4) SIJ shear test, (5) tenderness of the PSIS, and (6) tenderness of the STL. SIJ pain was definitively diagnosed when there was $\geq 70 \%$ relief in pain in the SIJ region post treatment with fluoroscopic guided injections ${ }^{8,9)}$.

Diagnosis of pain originating from the STL: When patients indicated the lower-buttock around the ischial tuberosity as the location of pain (Fig. 1), we evaluated the bone-ligament attachment site of the STL (ischial tuberosity attachment site) for tenderness under ultrasound guidance (Fig. 2). Strength of $4 \mathrm{~kg}$ weight was applied on the tender point using the thumb $^{10)}$. The presence of tenderness was measured twice at the same site, with positive results both times being considered positive. If the pain was reproduced on needle insertion under fluoroscopic guidance, $2 \mathrm{~mL}$ local anesthetic ( $1 \%$ lidocaine) was administered at the site. A definitive diagnosis of pain originating from the STL was established when pain relief of $>70 \%$ was achieved by the injections after 15 min (Fig. 3).

Measurement of SLR difference, BF tenderness, and GM strength: The angle between the legs during passive straight leg
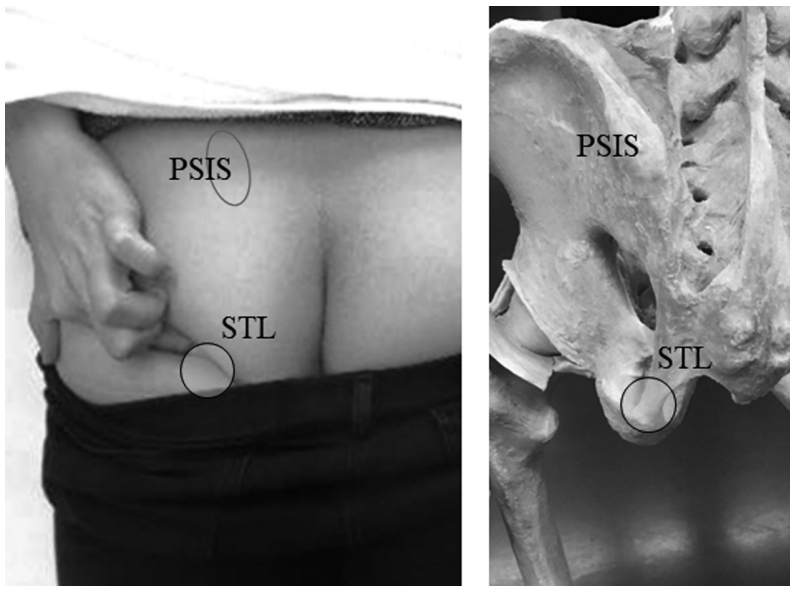

Fig. 1. Identify subjective pain area using the index finger (onefinger test). If the index finger is indicated around the ischial tuberosity, the painful area is confirmed by ultrasound.

PSIS: posterior superior iliac spine, STL: sacrotuberous ligament.

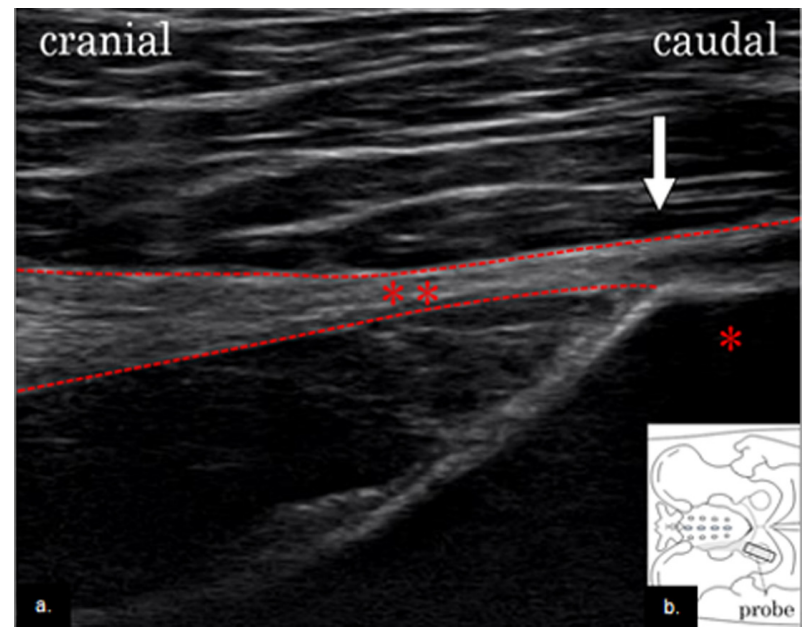

Fig. 2. Identification of the sacrotuberous ligament. The sactorubetous ligament (dotted line) is confirmed on basis of probe compression test by using ultrasound.

a. Ultrasound image, ischial tuberosity $(*)$, sacrotuberous ligament $(* *)$, tender point (white arrow), b. probe position. 
raising (SLR difference) was calculated by subtracting the test result (i.e., the angle on the affected side) from the SLR angle on the non-affected side. Tenderness of the BF (1/3 distal part), the site of tenderness in the $1 / 3$ distal part of the lateral thigh was identified as BF using ultrasound (Fig. 4). Strength of $4 \mathrm{~kg}$ weight was applied to the tender point using the thumb ${ }^{10)}$.

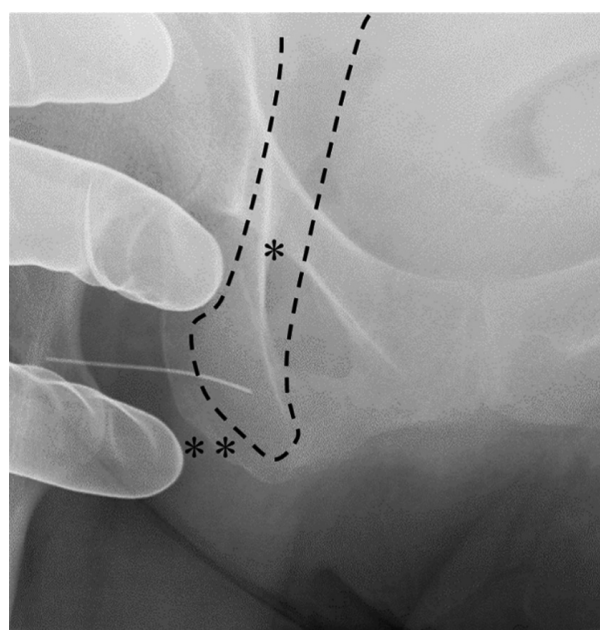

Fig. 3. Injection into the sacrotuberous ligament (*: dotted line). Pain reproduction is confirmed by needle insertion at the ischial tuberosity $(* *)$ of the sacrotuberous ligament.

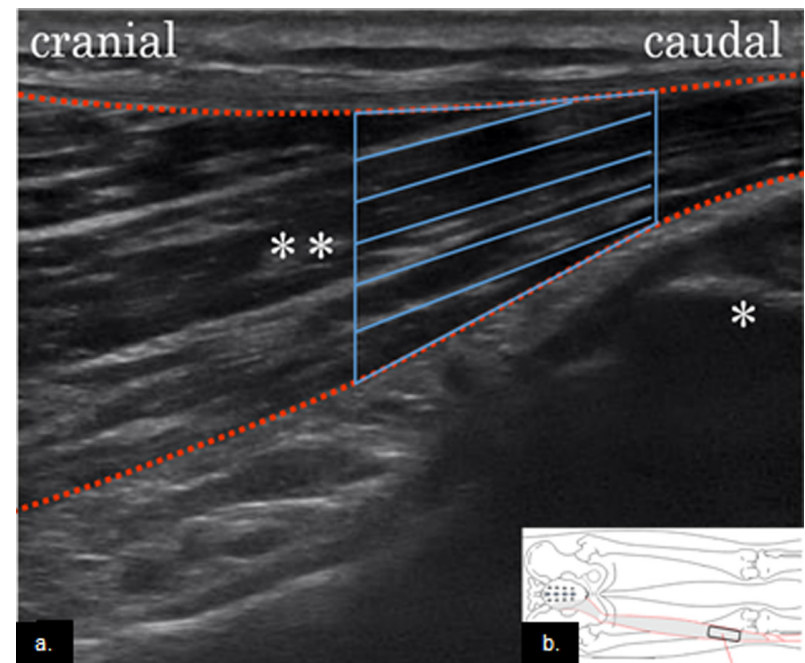

Fig. 4. Biceps femoris muscle (dotted line), confirmed by ultrasound. An ultrasound was used at a site where tenderness (shaded area) could be confirmed, and the probe compression test was positive and identified as biceps femoris muscle.a. Ultrasound image, femur $(*)$, biceps femoris muscle $(* *)$, tender point (shaded area), b. probe position

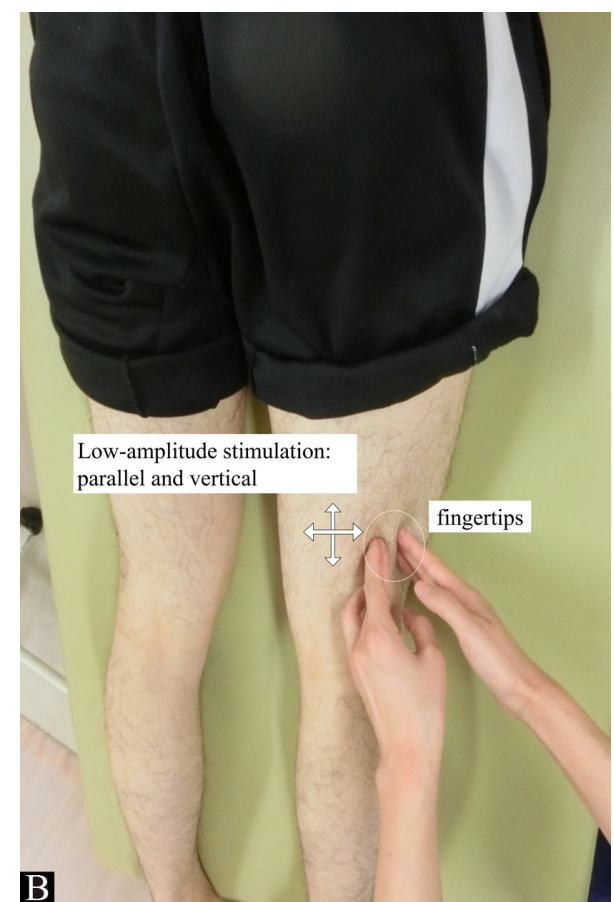

Fig. 5. A. Physical therapy technique. Relaxation of the sacrotuberous ligament. Hold the sacrotuberous ligament (the ischial tuberosity side) with one hand while sliding the other hand back and forth in small motions to first relax the sacrotuberous ligament and then stretch it. B. Physical therapy technique. Relaxation of the biceps femoris muscle. Biceps femoris muscle relaxation was performed on the tender area, and relaxation was performed using bilateral fingertips with low-amplitude stimulation parallel and vertical to the fibrous direction under pressure. 
The GM strength was measured using manual muscle testing (MMT).

Physical therapies were conducted as follows. Relaxation of the STL was performed first. BF relaxation and GM contraction exercises were added, if necessary. The following list sets out the physical therapy techniques used.

The STL relaxation (the ischial tuberosity side) was held with one hand while sliding the other hand back and forth in small motions to first relax and then stretch the STL (Fig. 5A). BF relaxation was performed at the tender area using bilateral fingertips movements with low-amplitude stimulation parallel and vertical to the fibrous direction under pressure (Fig. 5B). GM contraction exercises were carried out in the prone position, starting with active assistive exercises, followed by active and resistance exercises.

Assessment of the effectiveness of physical therapy for pain at the STL: The patients were asked to report on the intensity of persisting STL pain after one physical therapy session based on the assumption that the pain before the procedure was quantified as level 10. It was considered that the physical therapy option was effective when patients reported the intensity of persisting STL pain just after the procedure as less than level 3, which indicated an improvement of at least $70 \%$. Physical therapy sessions continued until STL pain was relieved sustainably by at least $70 \%$, i.e., the pain did not reoccur.

Statistical analysis was performed as follows. The patients were categorized into three groups based on physical therapeutic options that proved effective on them: improvement after STL relaxation (STL group); improvement after BF and STL relaxation (BF group); and improvement after GM contraction and STL relaxation exercise (GM group). The SLR difference and GM strength were compared between the three groups using the Kruskal-Wallis test and post hoc Steel-Dwass test. The differences in BF tenderness were analyzed using Fisher's exact test with Bonferroni correction. A p-value of less than 0.05 was considered statistically significant. All the statistical analyses were conducted with EZR (version 3.3.2) —a statistical software that extends the capabilities of $\mathrm{R}$ and $\mathrm{R}$ Commander.

\section{RESULTS}

The incidence of sacrotuberous ligament pain in the persisting lower-buttock pain was $57.9 \%$ (33 out of 57). Of these 33 patients with residual STL pain, 11 patients (33.3\%) experienced sustained pain relief after STL injection alone. The remaining 22 patients $(66.7 \%)$ needed physical therapy in addition to injections. Physical therapy helped eight patients improve with STL relaxation alone (STL group). BF and STL relaxation were effective in the case of eight patients (BF group). Finally, the GM contraction and STL relaxation exercises were effective in the case of the six patients (GM group) (Fig. 6).

The BF group exhibited both higher SLR difference and frequency of significant BF tenderness compared to the other two groups $(\mathrm{p}<0.05)$. The GM group showed significantly lower GM strength compared to the other two groups $(\mathrm{p}<0.05)$. All patients in the STL group did not have SLR differences, BF tenderness, and weakness of GM strength (Table 1). Table 2 shows the number of physical therapy sessions and time taken to recover from STL pain. The GM group needed more sessions and longer time to recover than the other groups.

\section{DISCUSSION}

SIJ-related ligaments, including the STL, contribute to stabilizing the SIJ. The STL tension could change owing to a minor subluxation of the SIJ, particularly at the adhesion part of the STL. Therefore, STL pain can occasionally be associated with SIJ dysfunction and is expected to be relieved after SIJ treatments. However, in this study, we found that 57 out of 303 patients with SIJ dysfunction showed persisting lower-buttock pain even after SIJ treatments, and more than half of the patients had residual STL pain.

Here, STL tenderness was assessed under ultrasound guidance and confirmed the pain originating from the STL using local anesthetic injections under fluoroscopic guidance. Several studies on the STL, which could be a cause of lower-buttock pain, have been conducted ${ }^{4-7}$. Prolotherapy ${ }^{4)}$ and positional release ${ }^{7)}$ were also reported as the treatment options for STL pain, but a method to diagnose the pain definitively has not been established. We believe that the diagnostic methods used here are rigorous and the results reliable.

Regarding STL pain management, the local anesthetic injection used for the purpose of diagnosis itself provided sustained pain relief in one-third of the patients. However, the other two-thirds of the patients required additional physical therapy because of recurred STL pain even after the injections.

This study revealed several key physical findings for choosing options for treating STL pain. STL relaxation alone was effective for patients without the restriction of their SLR on the affected side compared to the non-affected side, BF tenderness, and GM weakness. Additional BF relaxation was needed when patients exhibited restricted SLR on the affected side and high BF tenderness (1/3 distal part). The STL connects the sacral lateral bulge (S3-S5) and the ischial tuberosity, and it restricts the nutation motion of the sacrum ${ }^{11)}$ (Fig. 7A). The BF connects the distal portion of the ischial tuberosity to the fibular head. The proximal portion of the BF also connects to the surface fibrils of the STL ${ }^{12}$, 13). Therefore, the BF contraction induces tilting of the pelvis, limits the anterior tilt of the pelvis ${ }^{14)}$, and affects the tension in the STL ${ }^{15}$ ) (Fig. 7B). Pain at the STL attachment site without contracture of the BF could be resolved by STL relaxation alone after an improvement of the SIJ dysfunction. However, in patients with contracture of the BF, BF and STL relaxation (1/3 distal part) are necessary to alleviate STL pain even after an improvement of the SIJ dysfunction. 
Additional GM contraction exercise was needed for patients with GM weakness identified in the first physical assessment session. The origin of the GM is at the iliac crest, the lower part of the sacrum and coccyx, and insertion is at the posterior gluteal line ${ }^{16)}$. It connects to the iliotibial tract and gluteal tuberosity ${ }^{16)}$. The contraction of the GM could compress the SIJ and stabilize the joint ${ }^{5,17-20)}$ (Fig. 7C). GM weakness could affect the loosening of the SIJ, allowing excessive nutation of the sacrum and resulting in increased STL tension. Thus, the GM contraction exercise was effective in reducing STL pain.

These three kinds of physical therapy options were revealed to relieve the residual STL pain after treatment of the SIJ dysfunction. It was also found that recovery took the longest when there was muscle weakness in the GM in the first evaluation.

This study had several limitations. First, the number of cases in each group was small. Second, the reasons why BF contracture and GM weakness were observed could not be identified. These may relate to the duration of illness and/or medical history; however, we could not investigate this in detail. Third, the study considered treatment options for STL pain based on the results of both the first physical examination and the adopted treatment methods. Therefore, the present retrospective study provided a preliminary flowchart to choose treatment options. Prospective studies are required to confirm the usefulness of this flowchart. Despite its limitations, this study provided three kinds of physical therapy options for patients with STL pain.

From the above discussion, it could be concluded that the incidence of lower-buttock pain originating from the STL was $57.9 \%$ (33 out of 57). Two-thirds of patients with STL pain needed physical therapy. In addition to STL relaxation, BF relaxation was effective in patients who showed both higher SLR difference and BF tenderness, and GM contraction

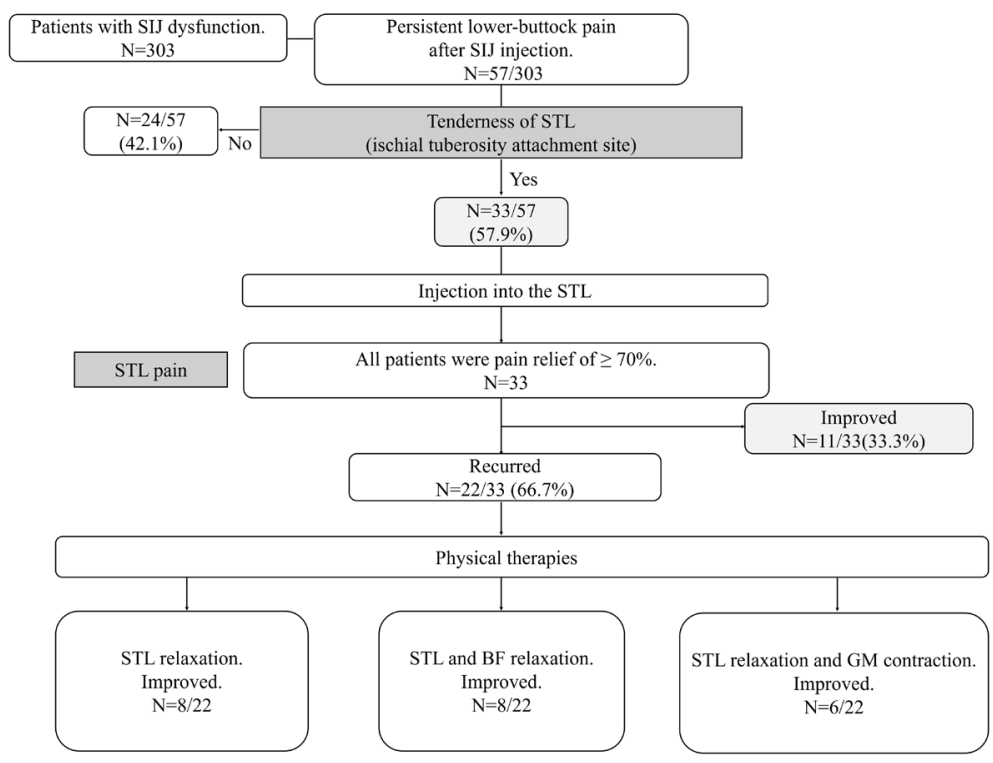

Fig. 6. Subject flow. Incidence and improvement rate of lower-buttock pain originating from the sacrotuberous ligament after treatment of sacroiliac joint dysfunction.

SIJ: sacroiliac joint, STL: sacrotuberous ligament, BF: biceps femoris muscle, GM: gluteus maximus muscle.

Table 1. Patient attributes and comparison of physical findings during the first intervention

\begin{tabular}{|c|c|c|c|c|c|c|}
\hline & \multirow{2}{*}{$\begin{array}{l}\text { STL group } \\
\quad n=8\end{array}$} & \multirow{2}{*}{$\begin{array}{c}\text { BF group } \\
n=8\end{array}$} & \multirow{2}{*}{$\begin{array}{c}\text { GM group } \\
\quad n=6\end{array}$} & \multicolumn{3}{|l|}{$\mathrm{p}$ value } \\
\hline & & & & STL vs. BF & STL vs. GM & BF vs. GM \\
\hline $\begin{array}{l}\text { SLR difference, } \\
\text { angle }\left(^{\circ}\right)\end{array}$ & $-2.5 \pm 3.8$ & $-20.6 \pm 5.6$ & $-5.0 \pm 6.3$ & 0.002 & 0.751 & 0.009 \\
\hline $\begin{array}{l}\text { BF tend. } \\
\text { (distal } 1 / 3 \text { part) }\end{array}$ & $0 / 8$ & $8 / 8$ & $0 / 6$ & $<0.001$ & 1.000 & 0.001 \\
\hline $\begin{array}{l}\text { GM. MMT, } \\
\text { median, range }\end{array}$ & $4(4-5)$ & $4(4-5)$ & $2(2-3)$ & 0.860 & 0.003 & 0.004 \\
\hline
\end{tabular}

median, range

n: number of patients; STL: sacrotuberous ligament; BF: biceps femoris muscle; GM: gluteus maximus muscle. SLR difference; angle between the legs during passive straight leg raising, mean (SD), BF tenderness (BF tend.); patient number of tenderness positive, GM. MMT; Manual muscle testing (MMT) assessment of GM strength.

Statistical method: Kruskal-Wallis with post hoc Steel-Dwass (SLR difference, GM. MMT); Fisher's exact test (BF tend.). 
exercises were effective in patients with GM weakness. These three kinds of physical therapy options were revealed to relieve the residual STL pain after treatment of the SIJ dysfunction.

\section{Conference presentation}

Part of the data of this study was presented in the 10th Interdisciplinary World Congress on Low Back and Pelvic Girdle Pain in 2019 .

Table 2. Number of sessions and treatment duration in each group

\begin{tabular}{lccc}
\hline & STL group & BF group & GM group \\
\hline $\begin{array}{l}\text { Number of sessions, } \\
\text { average, range }\end{array}$ & $7.6(3-24)$ & $7.9(2-24)$ & $21.2(9-52)$ \\
$\begin{array}{l}\text { Treatment duration (months), } \\
\text { average, range }\end{array}$ & $3.5(1.1-5.0)$ & $4.3(1.0-6.0)$ & $9.0(1.2-16.0)$ \\
\hline
\end{tabular}

STL: sacrotuberous ligament; BF: biceps femoris muscle; GM: gluteus maximus muscle.
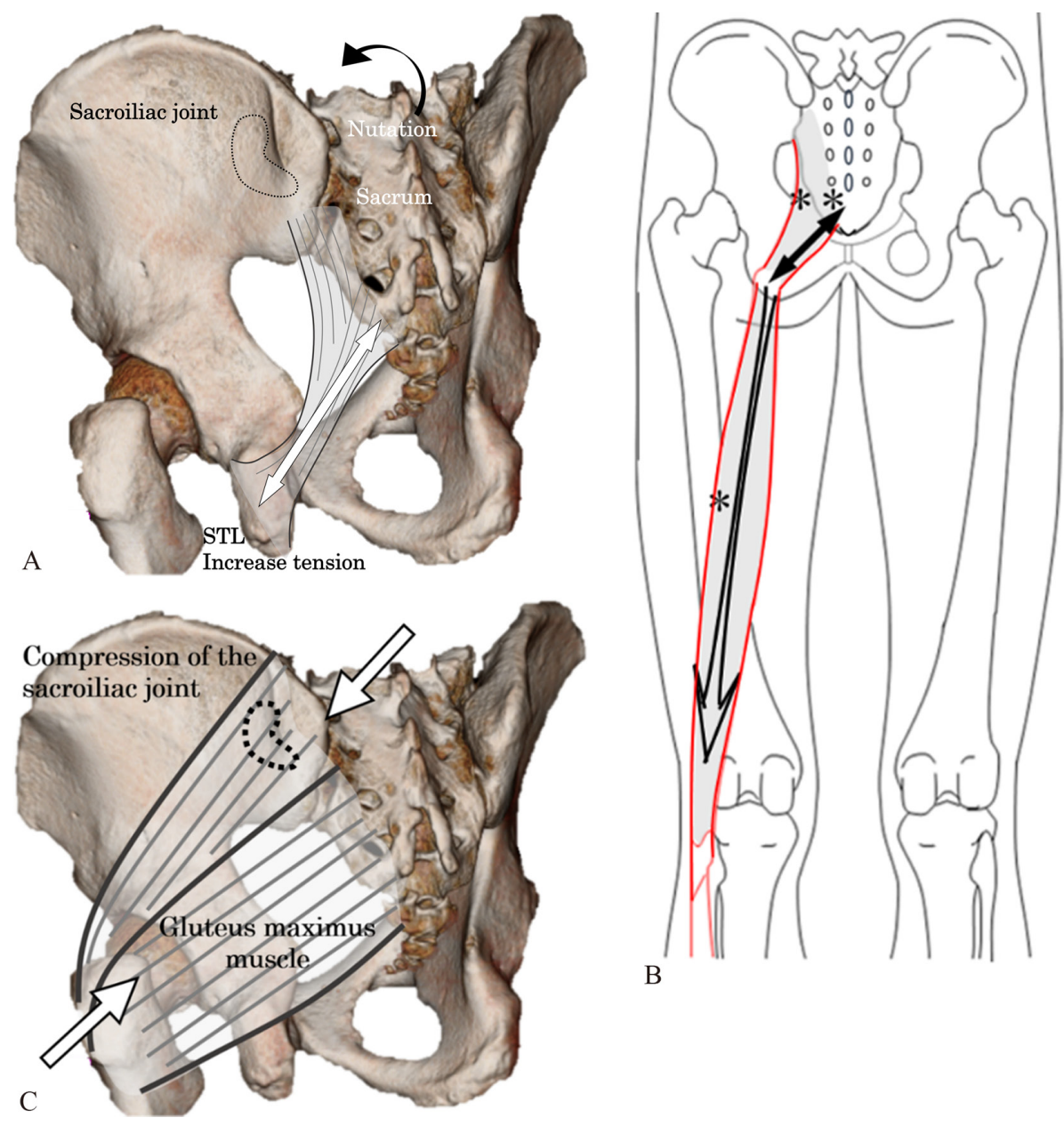

Fig. 7. A. Effects of sacrotuberous ligament, biceps femoris muscle, and gluteus maximus muscle on the sacroiliac joint. A; Effects of sacrotuberous ligament (black and gray lines) on the sacroiliac joint. Tension during nutation of the sacrum (black arrow), controlling the sacroiliac joint (dotted line), by increased the sacrotuberous ligament tension (white arrow).

STL: sacrotuberous ligament

B. Effects of biceps femoris muscle (BF) on the sacroiliac joint. Relationship between biceps femoris muscle and the sacrotuberous ligament. The tension (white arrow) in the biceps femoris $\left(^{*}\right)$ continues with the superficial fibers of the sacrotuberous ligament and is involved in tension (black arrow) of the sacrotuberous ligament $(* *)$.

C. Effect of the gluteus maximus (black and gray lines) on the sacroiliac joint. The sacroiliac joint (dotted line) is compressed and stabilized by the glueus maximus contraction (white arrow). 


\section{Funding}

None.

\section{Conflict of interest}

There are no conflicts of interest to declare.

\section{REFERENCES}

1) Kurosawa D, Murakami E, Ozawa H, et al.: A diagnostic scoring system for sacroiliac joint pain originating from the posterior ligament. Pain Med, 2017, 18: 228-238. [Medline]

2) Murakami E, Aizawa T, Noguchi K, et al.: Diagram specific to sacroiliac joint pain site indicated by one-finger test. J Orthop Sci, 2008, 13: 492-497. [Medline] [CrossRef]

3) Murakami E, Kurosawa D, Aizawa T: Treatment strategy for sacroiliac joint-related pain at or around the posterior superior iliac spine. Clin Neurol Neurosurg, 2018, 165: 43-46. [Medline] [CrossRef]

4) Hackett GS: Treated by prolotherapy. Ligament and tendon relaxation, 3rd ed. Springfield: Charles C Thomas Pub, 1958, pp 26, 45, 46, 62.

5) Vleeming A, Snijders C, Stoeckart R, et al.: The role of the sacroiliac joints in coupling between spine, pelvis, legs and arms. In: Movement, stability and low back pain. Edinburgh: Churchill Livingstone, 1997, pp 53-72.

6) Hauser RA, Hauser MA: Pain referral patterns from lumbosacral and pelvic joint ligament. Prolong your pain away, 2nd ed. Oak Park: Beulah Land Press, $2004, \mathrm{p} 46$.

7) Leon C, Judi D: The Lower body. Clinical application of neuromuscular techniques, 2nd ed. Edinburgh: Church Livingstone, 2011 , p 377, p 382.

8) Murakami E, Tanaka Y, Aizawa T, et al.: Effect of periarticular and intraarticular lidocaine injections for sacroiliac joint pain: prospective comparative study. J Orthop Sci, 2007, 12: 274-280. [Medline] [CrossRef]

9) Murakami E: Sacroiliac joint disorder: accurately diagnosing low back pain. Singapore: Springer, 2018, pp 34-35, pp 55-80.

10) Kurosawa D, Murakami E: Pelvic girdle tenderness points to differentiate the sacroiliac joint dysfunction from lumbar diseases. Orthop Surg Jpn, 2012, 63: 1231-1235 (in Japanese).

11) Vleeming A, Stoeckart R: The role of the pelvic girdle in coupling the spine and the legs: a clinical-anatomical perspective on pelvic stability. In: Movement, stability and lumbopelvic pain. Edinburgh: Churchill Livingstone, 2007, pp 113-134.

12) van Wingerden JP, Vleeming A, Snijders CJ, et al.: A functional-anatomical approach to the spine-pelvis mechanism: interaction between the biceps femoris muscle and the sacrotuberous ligament. Eur Spine J, 1993, 2: 140-144. [Medline] [CrossRef]

13) Philippon MJ, Ferro FP, Campbell KJ, et al.: A qualitative and quantitative analysis of the attachment sites of the proximal hamstrings. Knee Surg Sports Traumatol Arthrosc, 2015, 23: 2554-2561. [Medline] [CrossRef]

14) Gajdosik RL, Hatcher CK, Whitsell S: Influence of short hamstring muscles on the pelvis and lumbar spine in standing and during the toe-touch test. Clin Biomech (Bristol, Avon), 1992, 7: 38-42. [Medline] [CrossRef]

15) Woodley SJ, Kennedy E, Mercer SR: Anatomy in practice: the sacrotuberous ligament. NZ J Physio Ther, 2005, 33: 91-94.

16) Farfan HF: The biomechanical advantage of lordosis and hip extension for upright activity. Man as compared with other anthropoids. Spine, 1978, 3: 336-342. [Medline] [CrossRef]

17) Vleeming A, Wingerden van JP, Snijders CJ, et al.: Load application to the sacrotuberous ligament: influences on sacroiliac joint mechanics. Clin Biomech (Bristol, Avon), 1989, 4: 204-209. [CrossRef]

18) Vleeming A, Pool-Goudzwaard AL, Stoeckart R, et al.: The posterior layer of the thoracolumbar fascia. Its function in load transfer from spine to legs. Spine, 1995, 20: 753-758. [Medline] [CrossRef]

19) Vleeming A, Pool-Goudzwaard AL, Hammudoghlu D, et al.: The function of the long dorsal sacroiliac ligament: its implication for understanding low back pain. Spine, 1996, 21: 556-562. [Medline] [CrossRef]

20) Barker PJ, Hapuarachchi KS, Ross JA, et al.: Anatomy and biomechanics of gluteus maximus and the thoracolumbar fascia at the sacroiliac joint. Clin Anat, 2014, 27: 234-240. [Medline] [CrossRef] 